\title{
Genetic Variability Within and Among Mycelial Compatibility Groups of Sclerotium rolfsii in South Africa
}

\author{
A. J. Cilliers, L. Herselman, and Z. A. Pretorius
}

First and second authors: ARC Grain Crops Institute, Private Bag X1251, Potchefstroom 2520, South Africa; third author: Department of Plant Pathology, University of the Orange Free State, P.O. Box 339, Bloemfontein 9300, South Africa. Accepted for publication 5 May 2000.

\begin{abstract}
Cilliers, A. J., Herselman, L., and Pretorius, Z. A. 2000. Genetic variability within and among mycelial compatibility groups of Sclerotium rolfsii in South Africa. Phytopathology 90:1026-1031.

Isolates of Sclerotium rolfsii, the causal organism of stem rot or southern blight of groundnut, can be placed in mycelial compatibility groups (MCGs) based on hyphal interactions between isolates. The aim of this study was to determine whether amplified fragment length polymorphism (AFLP) analysis was a suitable technique to assess genetic variability between isolates and MCGs of $S$. rolfsii. For preliminary genetic analysis, 10 isolates were selected from each of two MCGs and compared with each other using the restriction enzymes EcoRI and MseI and 4 primer pairs. The number of polymorphisms ranged from 10 to 36 per
\end{abstract}

ABSTRACT primer combination, with an average of 22.5. AFLP analysis clearly showed genotypic differences (22\%) among MCGs B and C, with a maximum variation of $6.41 \%$ between any two isolates per group using four primer pairs. Certain isolates could not be distinguished from each other. A more in-depth study of 10 isolates from MCG B, using 8 additional primer pairs, showed small genetic differences (maximum of $4.2 \%$ and minimum of $0.2 \%$ ) between isolates. These results suggested that DNA could be pooled for comparison of MCGs. Pooled DNA from isolates within groups using 20 primer pairs confirmed differences between 9 MCGs. This technique effectively differentiated MCGs of $S$. rolfsii from each other and also detected differences between isolates within a single MCG.

Additional keywords: genetic fingerprinting.
Sclerotium rolfsii Sacc. causes disease in over 500 plant species throughout the world $(1,11)$. The fungus does not produce asexual spores and perpetuates as sclerotia, the primary inocula, on plant debris and in soil (12). The sexual state, Athelia rolfsii (Curzi) Tu \& Kimbrough, has been induced in the laboratory (13), but it is not thought to occur commonly in nature. S. rolfsii was first recorded in South Africa on tobacco in 1926 (9) and as the causal organism of stem rot or southern blight on groundnut in 1931 (2). Stem rot is considered a serious disease of groundnut in South Africa, where it occurs commonly in the Vaalharts and Viljoenskroon production areas. Yield losses amounting to \$66 million have been estimated in Georgia (University of Georgia Cooperative Extension Service estimates, 1988 through 1992), and losses of 5 to $10 \%$ of the total groundnut crop in Alabama have been attributed to this disease (15).

Punja and Grogan (13) showed that $S$. rolfsii isolates can be placed in mycelial compatibility groups (MCG) based on mycelial interactions. They identified 25 groups from 72 isolates. When isolates from the same MCG were paired, hyphae intermingled with little or no cell death, whereas isolates from different groups formed antagonistic zones that were accompanied by plasmolytic killing of hyphal cells. Nalim et al. (10) identified 25 MCGs among $S$. rolfsii isolates collected from Texas. Using molecular markers, they found that all isolates within an MCG produced identical patterns and that some MCGs shared the same patterns for the internal transcribed spacer (ITS) regions and 18-base oligonucleotide primer, NK2. Harlton et al. (4) screened a worldwide collection of $S$. rolfsii and found that isolates from the same geo-

Corresponding author: A. J. Cilliers; E-mail address: andre_c@ops1.agric.za

Publication no. P-2000-0705-02R

(C) 2000 The American Phytopathological Society graphic area or host could often be grouped in the same MCG. In some cases, however, widely diverse isolates could also be grouped in the same MCG. Variation in ITS regions revealed 12 subspecific groupings, some of which correlated with their MCGs (4).

More recently, Punja and Sun (14) paired 128 isolates of $S$. rolfsii from 36 host species and 23 geographic regions and detected 68 MCGs. A comparison of these isolates by means of random amplified polymorphic DNA (RAPD) polymerase chain reaction amplifications showed no clear relationship between the host of origin and MCG, except that many isolates from the same host belonged to the same MCG. Conversely, isolates in a specific MCG could have originated from many different hosts.

Molecular markers are increasingly being used to characterize populations of plant pathogens (6,7). According to Majer et al. (5) molecular markers may be used to evaluate levels of genetic diversity and phylogenetic relationships within and between species and to identify particular races and pathotypes. Several different types of markers have been developed. Isozyme markers are relatively inexpensive and easy to use but tend to reveal low levels of polymorphisms in pathogenic fungi. RFLP (restriction fragment length polymorphism) markers may be highly informative if appropriate DNA probes are available, but in several studies, little variation was revealed. RAPD markers, although widely used, have different levels of success and suffer from lack of reproducibility (5). Majer et al. (5) suggested that AFLP (amplified fragment length polymorphism) fingerprinting is more suitable for detecting polymorphisms among fungal isolates, e.g., variability is assessed at a large number of independent loci, the markers are "neutral" (i.e., not subject to natural selection), variation can be revealed in any part of the genome, and data are reproducible and obtained quickly.

The objective of our study was to determine whether AFLP analysis can be used to differentiate among South African isolates and MCGs of S. rolfsii. 


\section{MATERIALS AND METHODS}

Collection of isolates. Isolates were collected from various host plants showing symptoms of $S$. rolfsii infection at different locations in South Africa during 1997 and 1998 (Table 1). One to three sclerotia were taken from each diseased plant and transferred to water agar (WA) dishes. Pure isolates were obtained using the hyphal tip technique and permanent cultures were established on potato dextrose agar (PDA) plates. Cultures were stored on PDA slants at $4^{\circ} \mathrm{C}$.

MCGs. All isolates were paired against each other in $100 \times 15-\mathrm{mm}$ PDA dishes to observe possible mycelial interactions as described by Punja and Grogan (13). Each pairing was performed twice, and all isolates were paired with themselves as controls. Six isolates were paired simultaneously and placed in different MCGs based on the presence of an antagonistic zone between the isolates.

DNA isolation. Isolates selected for AFLP analysis were cultured at $25^{\circ} \mathrm{C}$ in a nutrient-broth liquid medium that had been enriched with $16 \mathrm{~g}$ of glucose per liter. Each isolate was cultured in $20 \mathrm{ml}$ of medium in a 100-ml Erlenmeyer flask. After 2 weeks the mycelial mat was removed and freeze-dried. DNA was isolated using a modified version of the method described by Graham et al. (3). Approximately $750 \mu 1$ of CTAB buffer $(100 \mathrm{mM}$ Tris [pH 8.0], $20 \mathrm{mM}$ EDTA [pH 8.0], $1.4 \mathrm{mM} \mathrm{NaCl}, 2 \%$ CTAB [hexadecyltrimethylammonium bromide], and $0.2 \% \quad \beta$ mercapthoethanol) were added to $250 \mu 1$ of the freeze dried mycelium that had been ground to a fine powder. The suspension, contained in a $1.5-\mathrm{ml}$ microfuge tube, was thoroughly mixed and incubated at $65^{\circ} \mathrm{C}$ for $1 \mathrm{~h}$. Five hundred microliters of chloroform/isoamyl alcohol (24:1) was added, and the suspension was mixed by gentle inversion. After centrifugation at $12,000 \times g$ for $3 \mathrm{~min}$, the upper aqueous layer was transferred to a fresh tube containing $500 \mu \mathrm{l}$ of isopropanol, mixed by gentle inversion and incubated at room temperature for $20 \mathrm{~min}$. The mixture was centrifuged at $12,000 \times g$ for $5 \mathrm{~min}$ before adding $500 \mu 170 \%$ (wt/vol) of ethanol and incubated at room temperature for $20 \mathrm{~min}$. DNA was precipitated at $12,000 \times g$ for $5 \mathrm{~min}$, and the pellet was air-dried

TABLE 1. Location, host, number of South African isolates of Sclerotium rolfsii studied, and designated mycelial compatibility group (MCG)

\begin{tabular}{llcl}
\hline Location $^{\mathrm{a}}$ & \multicolumn{1}{c}{ Crop $^{\mathrm{b}}$} & No. of isolates $^{\mathrm{c}}$ & $\mathrm{MCG}^{\mathrm{d}}$ \\
\hline Potchefstroom & Arachis hypogaea & 22 & $\mathrm{~B}, \mathrm{E}$ \\
Potchefstroom & Helianthus annuus & 5 & $\mathrm{~B}, \mathrm{D}$ \\
Potchefstroom & Lupinus albus & 8 & $\mathrm{~B}, \mathrm{C}$ \\
Lichtenburg & A. hypogaea & 7 & $\mathrm{~A}$ \\
Viljoenskroon & A. hypogaea & 4 & $\mathrm{E}$ \\
Barkley West & A. hypogaea & 2 & $\mathrm{C}$ \\
Bosbokrand & A. hypogaea & 4 & $\mathrm{D}$ \\
Brits & A. hypogaea & 1 & $\mathrm{C}$ \\
Vaalharts & A. hypogaea & 2 & $\mathrm{~B}$ \\
Makathini & A. hypogaea & 3 & $\mathrm{~F}$ \\
Makathini & H. annuus & 5 & F,G \\
George & Daucus carota & 1 & $\mathrm{H}$ \\
Bergville & Glycine max & 9 & $\mathrm{I}$ \\
\hline
\end{tabular}

${ }^{a}$ Location in South Africa from which isolates were obtained.

${ }^{\mathrm{b}}$ Crop plants from which isolates were obtained.

${ }^{c}$ Total number of isolates collected per specific host and location.

${ }^{\mathrm{d}}$ Mycelial compatibility grouping. for $1 \mathrm{~h}$ and resuspended in TE buffer (10 mM Tris-Cl [pH 8.0] $1 \mathrm{mM}$ EDTA [pH 8.0]). The resuspended DNA was extracted with 0.1 volume of $7.5 \mathrm{M}$ ammonium acetate and an equal volume of chloroform/isoamyl alcohol (24:1). The aqueous layer was transferred to a fresh tube containing 2 volumes of cold absolute ethanol. The precipitated DNA was washed three times in cold $70 \%$ ethanol, and the pellet was air-dried and resuspended in TE buffer. The DNA was treated with RNase for $2 \mathrm{~h}$ at $37^{\circ} \mathrm{C}$. Concentration and purity of DNA were estimated by measuring absorbances at $A_{260}$ and $A_{280} \mathrm{~nm}$. DNA samples were diluted to a working solution of $200 \mathrm{ng} / \mu \mathrm{l}$.

AFLP analysis. AFLP analysis was performed following the protocol described by Vos et al. (16) and the product manual supplied by Life Technologies Inc. (Gaithersburg, MD), with minor modifications. Restriction enzymes EcoRI and $M s e I$ were used to digest $500 \mathrm{ng}$ of $S$. rolfsii genomic DNA per $\mu \mathrm{l}$ for $4 \mathrm{~h}$, and the reaction mix, without inactivation of the restriction endonucleases, was subjected to overnight ligation of adapters at $37^{\circ} \mathrm{C}$, followed by preamplification. The ligation mix was not diluted prior to preamplification, and the preamplification DNA was diluted only 1:5 prior to selective amplification. The selective amplification was conducted using two primers. The EcoRI primer contained two selective nucleotides and the $M s e I$ primer contained three, corresponding to the EcoRI and MseI linkers obtained from Life Technologies.

Gel electrophoresis was performed as described by Vos et al. (16) and modified by employing a 5\% denaturing polyacrylamide gel (19:1 acrylamide/bis-acrylamide, $7 \mathrm{M}$ urea, $1 \times$ TBE buffer). Electrophoresis was performed at a constant $80 \mathrm{~W}$ for $2 \mathrm{~h}$. For DNA visualization, AFLP gels were silver-stained following the protocol described by the Silver Sequence DNA Sequencing System manual (Promega Corp., Madison, WI).

A total of 64 primer combinations $(8 \times 8)$ are available in the Life Technologies AFLP kit. To establish whether this technique could differentiate between MCGs of $S$. rolfsii, 4 primer-pair combinations were tested on 10 isolates from each of 2 randomly selected MCGs. The EcoRI primer E-AA (randomly selected) was used in combination with the following four possible MseI primers: M-CAA, M-CAC, M-CAG, and M-CAT (randomly selected). An additional eight primer pairs (Table 2) were tested on 10 isolates from $1 \mathrm{MCG}$ to determine the variation within a

TABLE 3. Number of isolates of Sclerotium rolfsii used for pooled DNA analysis from nine mycelial compatibility groups (MCGs)

\begin{tabular}{lcr}
\hline $\mathrm{MCG}^{\mathrm{a}}$ & No. of isolates $^{\mathrm{b}}$ & \multicolumn{1}{c}{ Isolate numbers $^{\mathrm{c}}$} \\
\hline $\mathrm{A}$ & 5 & $64,66,67,68,69$ \\
$\mathrm{~B}$ & 5 & $41,48,60,61,135$ \\
$\mathrm{C}$ & 5 & $10,19,21,24,121$ \\
$\mathrm{D}$ & 5 & $54,123,124,125,126$ \\
$\mathrm{E}$ & 4 & $89,106,109,142$ \\
$\mathrm{~F}$ & 5 & $87,117,137,141,144$ \\
$\mathrm{G}$ & 1 & 81 \\
$\mathrm{H}$ & 1 & 37 \\
$\mathrm{I}$ & 5 & $70,80,115,130,132$ \\
\hline
\end{tabular}

${ }^{a}$ Nine MCGs of $S$. rolfsii in South Africa.

${ }^{\mathrm{b}}$ Number of isolates used per MCG in the amplified fragment length polymorphism (AFLP) analysis.

c Isolates used in the AFLP analysis.

TABLE 2. Primer pairs tested on pooled DNA from nine mycelial compatibility groups of Sclerotium rolfsii ${ }^{\mathrm{a}}$

\begin{tabular}{|c|c|c|c|c|c|c|c|c|}
\hline & $\mathrm{M}^{\mathrm{b}}$-CAA & M-CAC & M-CAG & M-CAT & M-CTA & M-CTC & M-CTC & M-CTT \\
\hline $\mathrm{E}^{\mathrm{c}}-\mathrm{AA}$ & $\mathrm{x}$ & $\mathrm{x}$ & $\mathrm{x}$ & $\mathrm{x}$ & $\mathrm{x}$ & $\mathrm{x}$ & $\mathrm{x}$ & $\mathrm{x}$ \\
\hline E-AC & $\mathrm{x}$ & $\mathrm{x}$ & $\mathrm{x}$ & $\mathrm{x}$ & & & & \\
\hline E-TG & & & & & $\mathrm{x}$ & $\mathrm{x}$ & $\mathrm{x}$ & $\mathrm{x}$ \\
\hline
\end{tabular}

a Pairs tested are indicated with an $\mathrm{x}$.

${ }^{\mathrm{b}}$ MseI primers with three selective nucleotides used in amplified fragment length polymorphism (AFLP) analysis.

${ }^{\mathrm{c}}$ EcoRI primers with two selective nucleotides used in AFLP analysis. 


\section{A B C D E F G H I}
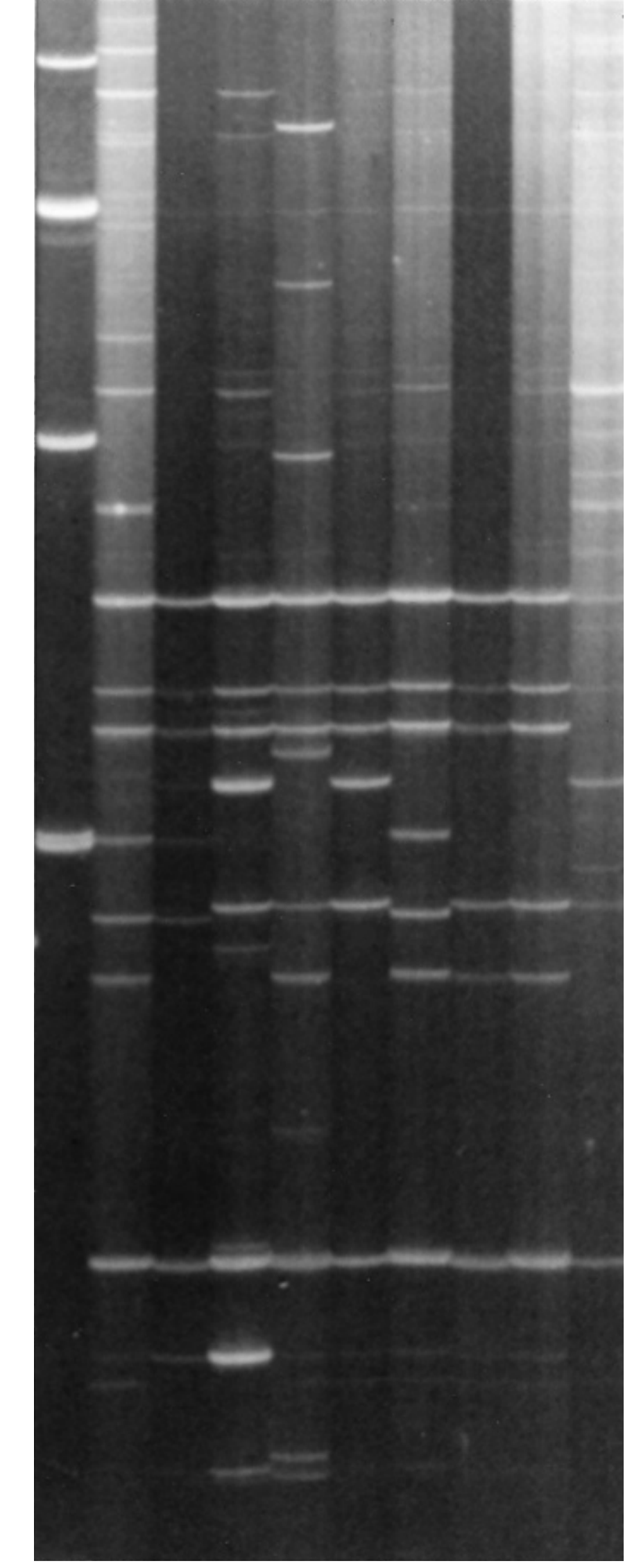

Fig. 1. Amplified fragment length polymorphism (AFLP) fingerprints of nine mycelial compatibility groups (MCGs) of Sclerotium rolfsii using the E-AA (EcoRI) and M-CAG (MseI) primer pair combination. Lane M, 100 bp molecular marker. MCGs A through I are designated as in Table 1. single group. DNA from various isolates (Table 3) within an MCG was pooled to compare groups with each other. This was done based on the relatively low between-isolate variation (maximum of $4.2 \%$ and minimum of $0.2 \%$ ) observed when variation within MCG B was tested. This low variation indicated that a better representative sample for each group could be obtained by pooling the DNA. The pooled DNA included all possible variation within each group, providing a better representation of the genotype of each MCG. Twenty different primer pair combinations were used to determine the genetic variability between the total number of MCGs detected. Reactions with each of the 20 primer pair combinations were repeated at least twice in independent experiments. Only reproducible results were used in statistical analyses.

Data analysis. The DNA bands obtained for each isolate or group of isolates were scored based on their presence (1) or absence (0). Only reliable and repeatable bands were considered. Pairwise genetic distances were expressed as the complement of Nei and Li's $F$ statistic. Cluster analysis was done by the unweighted paired group method using arithmetic averages (UPGMA). All calculations were performed with the aid of the program NTSYSpc version 2.02i (Exeter Software, New York). The goodness-of-fit of phenograms was determined by computing a cophenetic value matrix using the COPH module of NTSYSpc and comparing this matrix with the SAHN tree matrix using the MXCOMP module. A cophenetic correlation of $r>0.9$ was considered a very good fit.

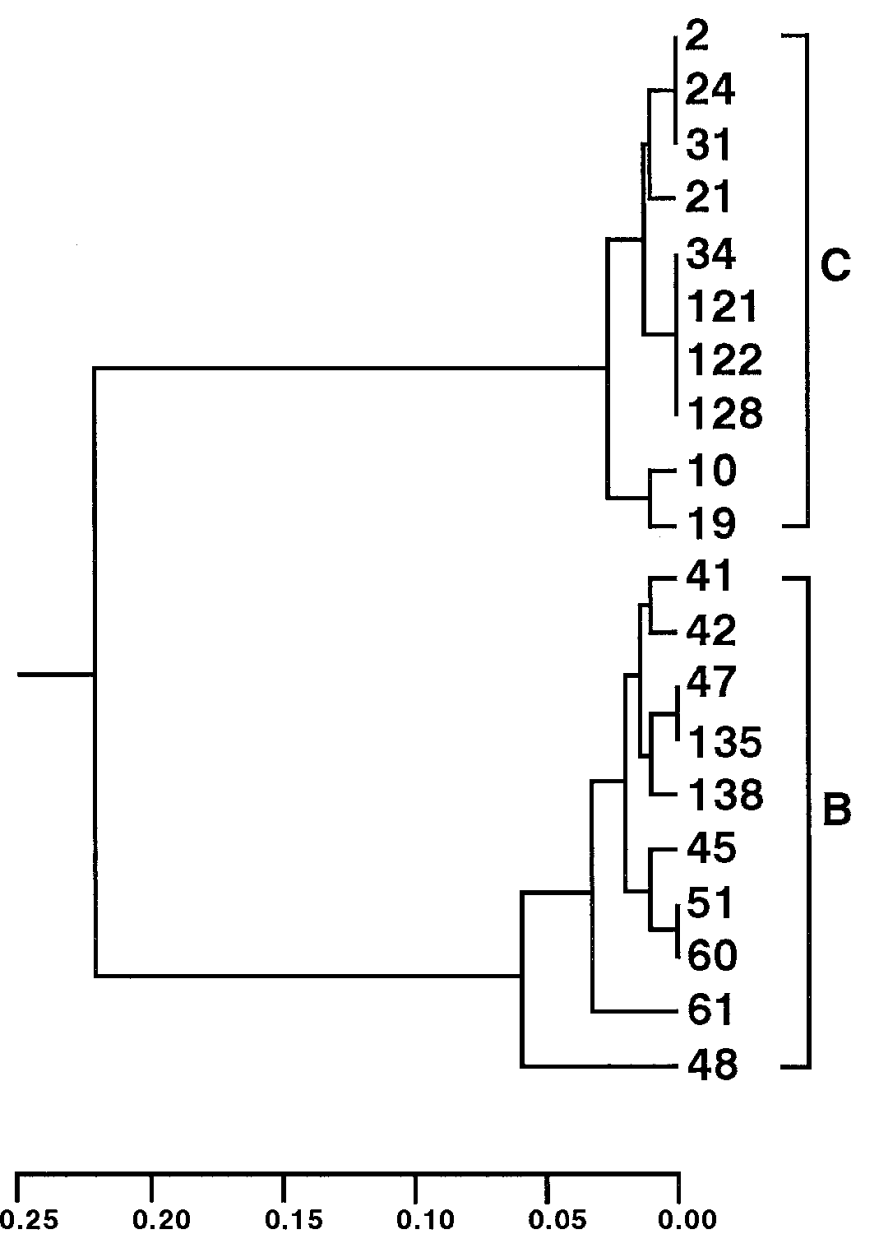

\section{Dissimilarity Coefficient}

Fig. 2. Phenogram showing the relationship among 10 isolates each of Sclerotium rolfsii mycelial compatibility groups (MCGs) B and C. Data were generated by scoring amplified fragment length polymorphism fingerprints after application of four primer pair combinations. 


\section{RESULTS}

Collection of isolates and MCGs. A total of 73 isolates of $S$. rolfsii was collected from 10 locations throughout South Africa. After pairing, all isolates could be assigned to one of nine MCGs which were designated with letters A through I. In certain cases, isolates from the same geographic area appeared to group together in the same MCG, but this was not true for isolates from the same host plant (Table 1). Some of the MCGs did, however, appear to be less host specific than others.

AFLP analysis. Polymorphisms among isolates of $S$. rolfsii were frequent and easy to score (Fig. 1). Primer combinations varied in their ability to detect polymorphisms, ranging from 10 to 36 polymorphisms per primer combination. On average a primer combination detected 22.5 polymorphisms. The total number of polymorphisms detected with the 20 primer combinations among the 9 groups was 449. A total of 668 fragments were amplified, with an average of 33.4 fragments per primer combination. Fragment sizes varied between 100 and 700 bps.

The AFLP analysis differentiated between MCGs B and C that were initially tested (Fig. 2). Clear differences were observed with each of the four primer pairs tested. The phenogram drawn using the pooled data of the four primer pairs showed a $22 \%$ dissimilarity between MCGs B and $\mathrm{C}$ and a maximum variation of $6.41 \%$ between isolates within an MCG. The obtained cophenetic correlation value of $r=0.985$ indicated that the UPGMA cluster analysis was statistically significant. Not all isolates could be distinguished from each other, since only four primer pairs were tested and the main objective was not to individually distinguish isolates from one another, but to determine the genetic diversity within an MCG.

The twelve primer pairs revealed smaller differences within MCG B than the differences which were observed between B and C. The phenogram showed maximum differences of $4.2 \%$ between the isolates (Fig. 3). A cophenetic correlation value of $r=$ 0.866 was obtained. The same general grouping was found between the isolates with these 12 primer pairs as was originally observed with the 4 primer pairs tested. The degree of relationship between the two phenograms was calculated by computing the cophenetic correlation, $r=0.759$, and the approximate Mantel $t$ test, with $t=3.417$. Most of the lack of fit between the two phenograms can be attributed to isolates 61 and 138. All 10 isolates could be distinguished from each other using 12 primer pairs. The largest observed difference between two isolates was only $4.2 \%$ providing justification for pooling DNA to investigate the variation between MCGs.

Using 20 primer pairs on the pooled DNA revealed clear differences between MCGs (Fig. 4). Differences between MCGs ranged from $9.3 \%$ between groups $\mathrm{A}$ and $\mathrm{F}$ to $34.1 \%$ between group D and the rest of the groups. A cophenetic correlation value of $r=0.823$ was obtained.

\section{DISCUSSION}

S. rolfsii has often been classified into MCGs $(10,13,14)$ and attempts have been made to understand the genetic structure of such populations (10). In this study, we applied AFLP fingerprinting to show genetic variation within and between nine MCGs of $S$. rolfsii detected in South Africa.

Based on the present study, it is clear that isolates within an MCG were genetically diverse (Fig. 3). Nalim et al. (10) studied isolates from groundnuts in Texas and found that isolates belonging to a specific MCG are clonal; their hypothesis was supported by similarities in morphology and host specificity. They placed $S$. rolfsii isolates in $25 \mathrm{MCGs}$, and the DNA amplification patterns resulting from the use of the 18-base oligonucleotide primer NK2 and from restriction digests of the ITS region of the rDNA were examined in a subset of 80 isolates representing 12 MCGs. Four of these MCGs were represented by only one isolate. Three NK2amplified DNA patterns in genomic DNA and four MboI restriction digest patterns of the ITS region were found. All isolates from an MCG produced identical patterns for each marker, and some MCGs shared the same ITS and NK2 patterns. Furthermore, MCGs having the same DNA patterns were often from the same field.

The similar DNA amplification patterns observed within MCGs (10) may be ascribed to the failure of the technique used to detect differences. In our study, AFLP fingerprints clearly differentiated among isolates within an MCG even though the groups appeared morphologically identical and often came from the same host plant. Although isolates from the same MCG (10 isolates each from groups B and C) differed from one another, they tended to cluster closer together, suggesting a smaller degree of genetic variability within a group and a larger degree of variability between groups (Fig. 2). The results from our study are consistent with those of Punja and Sun (14) in many respects. They found that MCGs clustered together genetically and that there was no correlation between geographic location and MCG. However, they did find isolates with identical RAPD patterns, which they hypothesized could be clonally derived. Among the isolates we tested, no two isolates had exactly the same banding pattern. Our data did not suggest the domination of one particular genotype on a given crop.

Harlton et al. (4) screened a worldwide collection of $S$. rolfsii isolates and identified 49 MCGs from 119 isolates. Isolates from the same geographic area or host often grouped in the same MCG, but in some cases, widely diverse isolates also grouped in the same MCG. Variation in the ITS regions was examined after restriction enzyme digests. Some of the isolates within an MCG showed different ITS-RFLP patterns, and certain patterns were also dispersed among MCGs. According to Harlton et al. (7) inter- and intraspecific variation within the ITS regions has been reported in several fungi and is mostly due to deletion or insertion events in
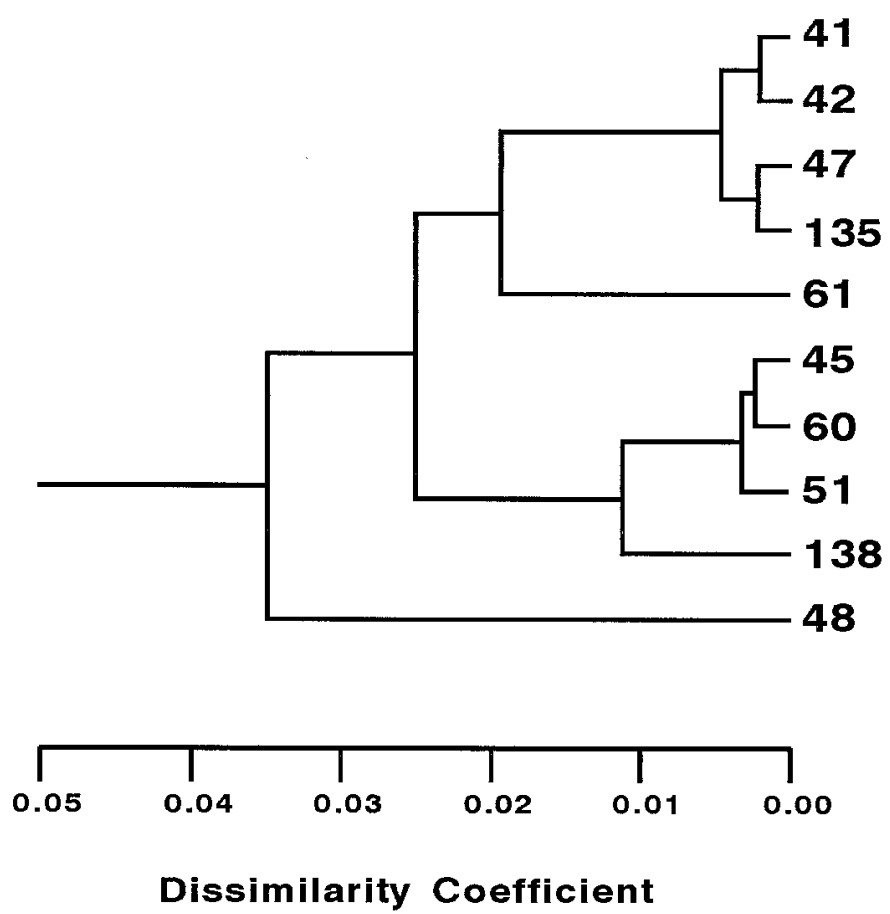

Fig. 3. Phenogram of 10 isololates of Sclerotium rolfsii mycelial compatibility group B. Data were generated by scoring amplified fragment length polymorphism fingerprints after application of 12 primer pair combinations. Bands were scored as present (1) or absent $(0)$, and a similarity matrix was derived using the SIMGEND program (NTSYSpc version 2.02i) using Nei's distance. A phenogram was reproduced by the unweighted pair group method for arithmetic average in the SAHN program. 
the ITS I and ITS II regions. Unique individuals are not necessarily correlated to the host nor are they restricted in geographic range (4). Clonally derived isolates within an MCG appear to share ITS restriction site similarity (4). In contrast, members within one MCG that are subject to different selection, mutation, and drift could possess the same vegetative compatibility alleles but possess different ITS sequences (4).

In our study, there was no apparent clustering according to host of origin or geographic area for the MCGs (Table 1). A cophenetic correlation value of $r=0.003$ indicated no correlation between cluster analysis based on genetic data and geographic data. The same was true $(r=0.194)$ for cluster analysis of host versus genetic data. However, MCGs containing isolates from more than one host and geographic area (groups B, C, and D) clustered separately from groups containing isolates from only one host or geographic area (Fig. 4). It therefore would appear that groups with a narrow host range are genetically dissimilar to those with a wider host range. Some of these MCGs, e.g., groups A and F, are more closely related genetically to each other and showed a smaller degree of genetic variation compared with others (Fig. 4). The same was true for individual isolates within an MCG, e.g., 41 and 42, 47 and 135, and 45 and 60 were closely related, respectively (Fig. 3), even though these isolates all came from different plants. Punja and Sun (14) showed that isolates within the same MCG were genetically diverse, as were isolates from the same geographic area. In their study, isolates from the same MCG however, did tend to group closer together, suggesting greater genetic similarity. The researchers concluded that isolates from widely distant geographic locations were more remotely related and isolates with identical RAPD patterns were in the same MCG and were probably clonally derived. We also observed, as did Harlton et al. (4), that isolates from one geographic area, e.g., Potchefstroom, frequently belonged to several MCGs (34 isolates were placed in four groups: B, C, D, and E). An MCG could also contain isolates from widely different geographic areas, e.g., group $\mathrm{C}$ contains isolates from Potchefstroom, Brits, and Barkley-Wes. However, in certain cases isolates from the same geographic area and host
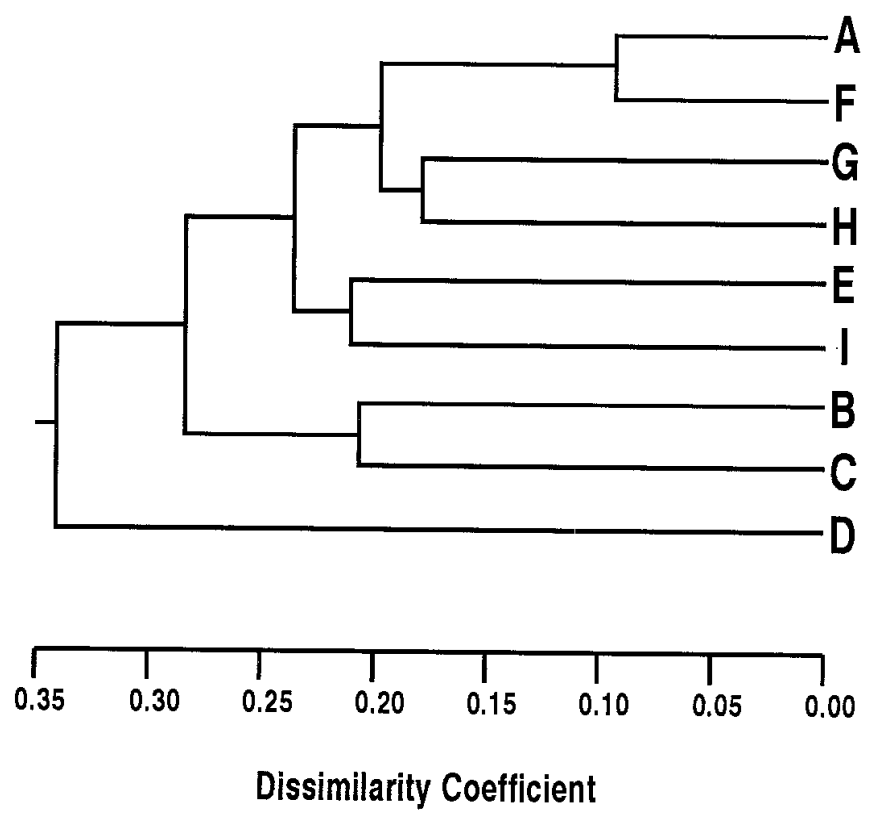

Fig. 4. Phenogram of nine mycelial compatibility groups of Sclerotium rolfsii. Data were generated by scoring amplified fragment length polymorphism fingerprints following application of 20 primer-pair combinations. Bands were scored as present (1) or absent (0) and a similarity matrix was derived using the SIMGEND program (NTSYSpc version 2.02i) using Nei's distance. A phenogram was reproduced by the unweighted pair group method for arithmetic average in the SAHN program. plant grouped together, e.g., isolates from groundnut in Lichtenburg all fell in group A.

The occurrence of the same MCG on different host species (e.g., group B) reflects the wide host range and spread of the pathogen. Also, the fact that isolates from within a given geographic area, e.g., Potchefstroom (group B), were diverse suggests that genetic changes had occurred within subpopulations. It may be possible, with further field studies, to link specific MCGs or groups of MCGs to pathogenicity on a particular crop plant. Such studies however, will require controlled pathogenicity tests and growth studies to determine whether virulence in the field varies for a specific crop plant.

Based on AFLP fingerprints, we conclude that genetic variation exists between $S$. rolfsii isolates of a single MCG. A larger degree of variation exists between MCGs, whether DNA from various isolates is pooled (Fig. 4) or not (Fig. 2). According to Milgroom (8), asexual reproduction results in offspring that are genetically identical to each other and their parent (or nearly so, but for mutation), the result of which is a clonal population structure. These clonal populations have distinctive features, such as widespread occurrence of identical genotypes, absence of recombinant genotypes, and correlations between independent sets of genetic markers. On the other hand, the meiotic phase associated with sexual reproduction results in independent assortment of chromosomes and recombination within chromosomes, causing greater genetic diversion (8). The sexual state of the fungus, Athelia rolfsii, is not thought to occur commonly in nature even though it has been induced in the laboratory (13). In the light of larger variation between MCGs and smaller variation between certain isolates within MCGs, we suggest that sexual and asexual reproduction may occur simultaneously. It is possible that sexual reproduction could explain the large differences observed between MCGs, while the smaller differences within MCGs could be attributed to more commonly occurring asexual reproduction. To verify this, however, the genome of a larger set of isolates in a given population will have to be tested.

\section{LITERATURE CITED}

1. Aycock, R. 1966. Stem rot and other diseases caused by Sclerotium rolfsii or the status of Rolf's fungus after 70 years. N.C. Agric. Exp. Stn. Tech. Bull. 174.

2. Doidge, E. M., and Bottomley, A. M. 1931. A revised list of plant diseases occurring in South Africa. Bot. Surv. S. Afr. Mem. 11.

3. Graham, G. C., Mayers, P., and Henry, R. J. 1994. Simple and rapid method for the preparation of fungal genomic DNA for PCR and RAPD analysis. Biotechniques 16:48-50.

4. Harlton, C. E., Lévesque, C. A., and Punja, Z. K. 1995. Genetic diversity in Sclerotium (Athelia) rolfsii and related species. Phytopathology 85:1269-1281.

5. Majer, D., Mithen, R., Lewis, B. G., Vos, P., and Oliver, P. R. 1996. The use of AFLP fingerprinting for the detection of genetic variation in fungi. Mycol. Res. 100:1107-1111.

6. McDonald, B. A. 1987. The population genetics of fungi: Tools and techniques. Phytopathology 87:448-453.

7. Michelmore, R. W., and Hulbert, S. H. 1987. Molecular markers for genetic analysis of phytopathogenic fungi. Annu. Rev. Phytopathol. 25:383404.

8. Milgroom, M. G. 1996. Recombination and the multilocus structure of fungal populations. Annu. Rev. Phytopathol. 34:457-477.

9. Moore, E. S. 1926. Diseases of Virginian tobacco in South Africa. Union S. Afr., J. Dep. Agric. 12:428-455.

10. Nalim, F. A., Starr, J. L., Woodard, K. E., Segner, S., and Keller, N. P. 1995. Mycelial compatibility groups in Texas peanut field populations of Sclerotium rolfsii. Phytopathology 85:1507-1512.

11. Punja, Z. K. 1985. The biology, ecology, and control of Sclerotium rolfsii. Annu. Rev. Phytopathol. 23:97-127.

12. Punja, Z. K. 1988. Sclerotium (Athelia) rolfsii, a pathogen of many plant species. Pages 523-534 in: Advances in Plant Pathology. Vol. 6, Genetics of Plant Pathogenic Fungi. G. S. Sidhu, ed. Academic Press, London.

13. Punja, Z. K., and Grogan, R. G. 1983. Hyphal interactions and antagonism among field isolates and single-basidiospore strains of 
Athelia (Sclerotium) rolfsii. Phytopathology 73:1279-1284.

14. Punja, Z. K., and Sun, L. J. 1997. Genetic diversity among mycelial compatibility groups of Sclerotiun rolfsii and Sclerotium delphini. Programme and summaries of the 11th biennial conference of the Australasian Plant Pathology Society, 29 September - 2 October 1997, Perth, Australia.
15. Sturgeon, R. V., Jr. 1986. Peanut disease loss estimates for major peanut producing states in the United States for 1984 and 1985. Proc. Am. Peanut Res. and Educ. Soc. 18:24-26.

16. Vos, P., Hogers, R., Bleeker, M., Reijans, M., van de Lee, T., Hornes, M. Frijters, A., Pot, J., Peleman. J., Kuiper, M. and Zabeau, M. 1995. AFLP: A new technique for DNA fingerprinting. Nucleic Acids Res. 21:4407-4414. 